\title{
Estratégias de enfermagem para prevenção de lesão por pressão em pacientes cirúrgicos
}

\author{
Nursing strategies for pressure injury prevention in surgical patients
Estrategias de enfermería para la prevención de lesiones por presión en pacientes quirúrgicos

\begin{abstract}
Sandra Marina Gonçalves Bezerra' 1, , Jessyca Fernanda Pereira Brito ${ }^{1}$, Jefferson Abraão Caetano Lira', Nanielle Silva Barbosa ${ }^{1}$, Kauan Gustavo de Carvalho', Luana Silva de Sousa ${ }^{1}$
\end{abstract}

ORCID IDs

Bezerra SMG (iD https://orcid.org/0000-0003-3890-5887

Brito JFP (D) https://orcid.org/0000-0003-1438-4650

Lira JAC (D) https://orcid.org/0000-0002-7582-4157

Barbosa NS (iD https://orcid.org/0000-0001-5758-2011

Carvalho KG (D) https://orcid.org/0000-0002-9940-1837

Sousa LS (D) https://orcid.org/0000-0002-2415-8334
COMO CITAR

Bezerra SMG; Brito JFP; Lira JAC; Barbosa NS; Carvalho KG; Sousa LS. Estratégias de enfermagem para prevenção de lesão por pressão em pacientes cirúrgicos. ESTIMA, Braz. J. Enterostomal Ther., 18: e1020, 2020. https://doi.org/10.30886/estima.v18.793_PT

\begin{abstract}
RESUMO
Objetivo: Identificar na literatura as estratégias utilizadas pela equipe de enfermagem para prevenção de lesão por pressão em pacientes cirúrgicos. Métodos: Revisão integrativa, utilizando a estratégia PICo, tendo como questão norteadora: "quais as estratégias utilizadas pela equipe de enfermagem para prevenção de lesão por pressão em pacientes cirúrgicos?" As buscas foram realizadas nas bases de dados Web of Science, MEDLINE via PubMed, CINAHL, Cochrane, Scopus e no índice bibliográfico LILACS via BVS, sendo analisados 12 estudos. Resultados: O Brasil se destacou com quatro publicações e os estudos de coorte, com nível de evidência IV, prevaleceram em seis artigos. As intervenções tecnológicas para prevenção de lesão por pressão no centro cirúrgico foram colchões para distribuição de pressão, dispositivo para redução de pressão no calcâneo, instrumentos validados específicos para a classificação do risco de lesão por pressão em pacientes cirúrgicos, além de superfícies de apoio contendo polímero viscoelástico e outros utensílios para alívio da pressão decorrente do peso e de dispositivos médicos. Com relação às intervenções educativas, destacaram-se os protocolos, os treinamentos e a simulação realística. Conclusão: Observou-se que essas estratégias reduziram a incidência de lesão por pressão, além de diminuírem os custos e garantirem a satisfação dos pacientes.
\end{abstract}

DESCRITORES: Período perioperatório. Período intraoperatório. Lesão por pressão. Posicionamento do paciente. Enfermagem. Estomaterapia.

\begin{abstract}
Objective: To identify in the literature the strategies used by nursing teams to prevent pressure injury in surgical patients. Methods: Integrative review, using the PICo strategy, with the guiding question: "What are the strategies used by the nursing team to prevent pressure injury in surgical patients?" The searches were performed in the Web of Science, MEDLINE via PubMed, CINAHL, Cochrane, Scopus and LILACS bibliographic index via VHL, from where 12 studies were analyzed. Results: Brazil stood out with four publications and cohort studies, with level of evidence IV, prevailed in six articles. Technological interventions for the prevention of Pressure Injury in the operating room were pressure distribution mattresses, a device to reduce pressure on the calcaneus, specific validated instruments for the classification of the risk of Pressure Injury in surgical patients, in addition to support surfaces containing polymer viscoelastic and other tools to relieve pressure from weight and medical devices. Regarding educational interventions, protocols, training and realistic simulation stood out. Conclusion: It was observed that these strategies reduced the incidence of pressure injury, in addition to reducing costs and ensuring patient satisfaction.
\end{abstract}

DESCRIPTORS: Perioperative period. Intraoperative period. Pressure ulcer. Patient positioning. Nursing. Enterostomal therapy.

1.Universidade Estadual do Piauí - Teresina (PI), Brasil.

*Autor correspondente: sandramarina20@hotmail.com

Recebido: Ago. 13, 2019 | Aceito: Mar. 31, 2020 


\section{RESUMEN}

Objetivo: identificar en la literatura las estrategias utilizadas por el equipo de enfermería para prevenir lesiones por presión en pacientes quirúrgicos. Métodos: Revisión integral, utilizando el acrónimo PICo, con la pregunta guía: “¿Cuáles son las estrategias utilizadas por el equipo de enfermería para prevenir lesiones por presión en pacientes quirúrgicos?" Las búsquedas se realizaron en la Web of Science, MEDLINE vía PubMed, CINAHL, Cochrane, Scopus y LILACS vía BVS, siendo 12 estudios analizados. Resultados: Brasil se destacó con cuatro publicaciones y estudios de cohortes, con nivel de evidencia IV, prevaleció en seis artículos. Intervenciones tecnológicas para la prevención de lesiones por presión en la sala de operaciones fueron colchones de distribución de presión, un dispositivo para reducir la presión sobre el calcáneo, instrumentos específicos validados para la clasificación del riesgo de lesión por presión en pacientes quirúrgicos, además de superficies de soporte que contienen polímeros de herramientas viscoelásticas y otras para aliviar la presión del peso y los dispositivos médicos. En cuanto a las intervenciones educativas, se destacaron los protocolos, la capacitación y la simulación realista. Conclusión: se observó que estas estrategias redujeron la incidencia de lesiones por presión, además de reducir los costos y garantizar la satisfacción del paciente.

DESCRIPTORES: Periodo perioperatorio. Periodo intraoperatorio. Úlcera por presión. Posicionamiento del paciente. Enfermería. Estomaterapia.

\section{INTRODUÇÃO}

A lesão por pressão (LP), caracterizada como um dano situado na pele e/ou em tecidos moles subjacentes, de maneira geral sobre uma proeminência óssea ou relacionada ao uso de dispositivos médicos ou outros artefatos, acomete o paciente cirúrgico devido à imobilidade prolongada ou pressão intensa, durante os procedimentos cirúrgicos, pois o efeito da anestesia bloqueia a sensibilidade à dor e à pressão excessiva. Nesse sentido, pressões maiores que $32 \mathrm{mmHg}$ resultam em uma oclusão do fluxo sanguíneo, propiciando isquemia tecidual e, por conseguinte, a $\mathrm{LP}^{1,2}$.

Paciente cirúrgico é aquele submetido a uma intervenção cirúrgica manual ou instrumental com o objetivo de diagnosticar ou tratar doenças. O processo cirúrgico é dividido em três fases: pré-operatório, intraoperatório e pósoperatório. A cirurgia segura é uma estratégia importante para reduzir a possibilidade de danos ao paciente e a prevenção de lesões de pele em pacientes cirúrgicos é um dos cuidados de enfermagem imprescindíveis ${ }^{3,4}$.

A transferência para sala cirúrgica, sala de recuperação pós-anestésica e clínica de origem aumenta a fricção e/ou o cisalhamento e, consequentemente, gera riscos para o surgimento de LP.Dessa forma, estratégias eficazes utilizadas pela equipe de enfermagem, a exemplo da educação em saúde, mudança de decúbito, uso de dispositivos e equipamentos para aliviar a pressão, são fundamentais para manter a segurança do paciente no centro cirúrgico e prevenir LP, além de garantir a qualidade do atendimento prestado ${ }^{5}$.

Estudo realizado em Minas Gerais, em 2018, constatou que o custo semestral do tratamento de LP por paciente internado foi de $\mathrm{R} \$ 1.886,00$, sendo que o custo total foi de
$\mathrm{R} \$ 113.186,00^{6}$. Nesse sentido, ressalta-se que as estratégias de prevenção de LP, apesar de apresentarem gasto considerável, possuem melhor custo benefício, uma vez que proporcionam redução da internação hospitalar, melhora da qualidade de vida e dos indicadores de prevalência e incidência de LP.

A incidência de LP no centro cirúrgico varia de 7,0 a $17,6 \%$, o que prolonga o tempo de internação, gera aumento dos custos hospitalares, assim como risco de infecção e outras consequências para os pacientes, profissionais e a instituição. A LP é a segunda reivindicação mais comum nos casos de morte por negligência, com 17.000 ações judiciais por ano, por ser 100\% evitável, destacando a importância de estratégias preventivas, uma vez que impactariam em uma economia considerada, cujos recursos poderiam ser utilizados para os outros fins, como a melhoria da qualidade da assistência ${ }^{7,8}$.

As LPs causam sofrimento emocional e físico, bem como dor severa, retardo na recuperação e alta hospitalar, o que predispõe o paciente a outras complicações ou mesmo ao óbito. Nessa perspectiva, cabe ao enfermeiro, no gerenciamento da assistência, o planejamento de estratégias eficazes para o adequado posicionamento do paciente e o uso dos equipamentos e dispositivos cirúrgicos, além da mobilização da equipe de enfermagem para a prevenção de LP'.

Este estudo tem como objetivo identificar na literatura as estratégias utilizadas pela equipe de enfermagem para prevenção de lesão por pressão em pacientes cirúrgicos.

\section{MÉTODOS}

Trata-se de uma revisão integrativa da literatura desenvolvida em seis etapas, seguindo o referencial teórico 
de Whittemore e Knaf ${ }^{10}: 1$ ) seleção da questão norteadora; 2) amostragem ou busca na literatura; 3) seleção das pesquisas que compuseram a amostra; 4) extração de dados dos estudos incluídos; 5) avaliação e interpretação dos resultados; e 6) apresentação da revisão ou síntese do conhecimento produzido.

A questão norteadora foi elaborada a partir do acrônimo PICo ${ }^{11}$, definindo-se $\mathrm{P}$ = população: "paciente cirúrgico",
I = interesse "estratégias de prevenção de lesão por pressão" e Co = contexto: "assistência de enfermagem”. Assim, elaborou-se a seguinte pergunta de pesquisa: "quais as estratégias utilizadas pela equipe de enfermagem para prevenção de lesão por pressão em pacientes cirúrgicos?”

Elencaram-se como critérios de inclusão artigos de estudos primários indexados em bases de dados, publicados

Tabela 1. Descritores controlados, não controlados e expressões de buscas utilizados para recuperação dos artigos. Teresina (PI), Brasil, 2020.

\begin{tabular}{|c|c|c|}
\hline \multicolumn{3}{|r|}{ MeSH e CINAHL } \\
\hline \multirow{2}{*}{$\mathrm{P}$} & DC & Perioperative period, Perioperative care, Intraoperative period \\
\hline & DNC & Perioperative period, Periods, perioperative, Perioperative care, Intraoperative period \\
\hline \multirow{2}{*}{1} & DC & Pressure ulcer, Patient positioning \\
\hline & DNC & Pressure ulcer, Pressure ulcers, Decubitus ulcer, Decubitus ulcers, Patient positioning \\
\hline \multirow{2}{*}{ Co } & DC & Nursing care, Nursing \\
\hline & DNC & Nursing care, Nursing, Nursings \\
\hline \multirow{7}{*}{ 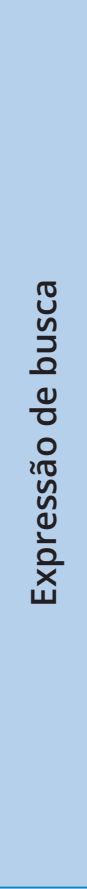 } & & 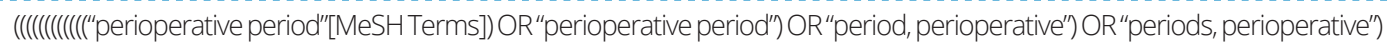 \\
\hline & $\begin{array}{l}\text { MEDLINE } \\
\text { via } \\
\text { Pubmed }\end{array}$ & 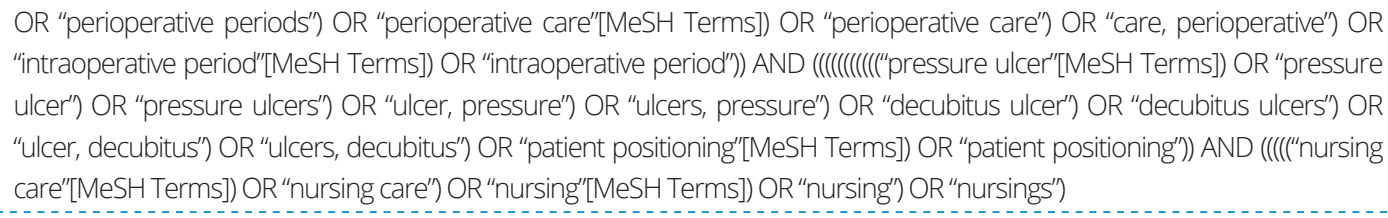 \\
\hline & $\begin{array}{l}\text { Web of } \\
\text { Science }\end{array}$ & $\begin{array}{l}\text { (TS =("perioperative period") OR TS=("perioperative periods") OR TS=("perioperative care") OR TS=("intraoperative } \\
\text { period")) AND (TS=("pressure ulcer") OR TS=("pressure ulcers") OR TS=("decubitus ulcer") OR TS=("decubitus } \\
\text { ulcers") OR TS=("patient positioning")) AND (TS=("nursing care") OR TS=(nursing) OR TS=(nursings)) }\end{array}$ \\
\hline & & ("perioperative period" OR (MH "Intraoperative Period") OR "perioperative periods" OR (MH "Perioperative Care") \\
\hline & CINAHL & $\begin{array}{l}\text { OR "perioperative care" OR (MH "Perioperative Care (lowa NIC)")) AND ((MH "Pressure Ulcer") OR "pressure ulcer" } \\
\text { OR "pressure ulcers" OR "decubitus ulcer" OR (MH "Patient Positioning") OR "patient positioning") AND ((MH } \\
\text { "Nursing Care") OR "nursing care" OR "nursing" OR "nursings") }\end{array}$ \\
\hline & Cochrane & $\begin{array}{l}\text { (("perioperative period") OR ("perioperative periods") OR ("perioperative care") OR ("intraoperative period")) AND } \\
\text { (("pressure ulcer") OR ("pressure ulcers") OR ("decubitus ulcer") OR ("decubitus ulcers") OR ("patient positioning")) } \\
\text { AND (("nursing care") OR (nursing) OR (nursings)) }\end{array}$ \\
\hline & Scopus & $\begin{array}{l}\text { ((TITLE-ABS-KEY ("perioperative period") OR TITLE-ABS-KEY ("perioperative periods") ORTITLE-ABS-KEY ("perioperative } \\
\text { care") OR TITLE-ABS-KEY ("intraoperative period"))) AND ((TITLE-ABS-KEY ("pressure ulcer") OR TITLE-ABS-KEY } \\
\text { ("pressure ulcers") OR TITLE-ABS-KEY ("decubitus ulcer") OR TITLE-ABS-KEY ("decubitus ulcers") OR TITLE-ABS-KEY } \\
\text { ("patient positioning"))) AND ((TITLE-ABS-KEY("nursing care")OR TITLE-ABS-KEY(nursing) OR TITLE-ABS-KEY (nursings))) }\end{array}$ \\
\hline \multicolumn{3}{|r|}{ DeCS } \\
\hline \multirow{3}{*}{$\mathrm{P}$} & DC & Período Perioperatório, Assistência Perioperatória, Período Intraoperatório \\
\hline & DNC & Período Perioperatório, Assistência Perioperatória, Cuidados Perioperatórios, Período Intraoperatório \\
\hline & DC & Lesão por Pressão, Posicionamento do Paciente \\
\hline I & DNC & $\begin{array}{l}\text { Lesão por Pressão, Úlcera de Decúbito, Úlcera de Pressão, Úlcera por Pressão, Úlceras por Pressão, } \\
\text { Posicionamento do Paciente }\end{array}$ \\
\hline \multirow{2}{*}{ Co } & DC & Cuidados de Enfermagem, Enfermagem \\
\hline & DNC & Cuidados de Enfermagem, Assistência de Enfermagem, Enfermagem \\
\hline 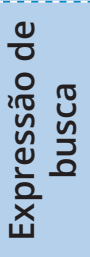 & $\begin{array}{l}\text { LILACS via } \\
\quad \text { BVS }\end{array}$ & 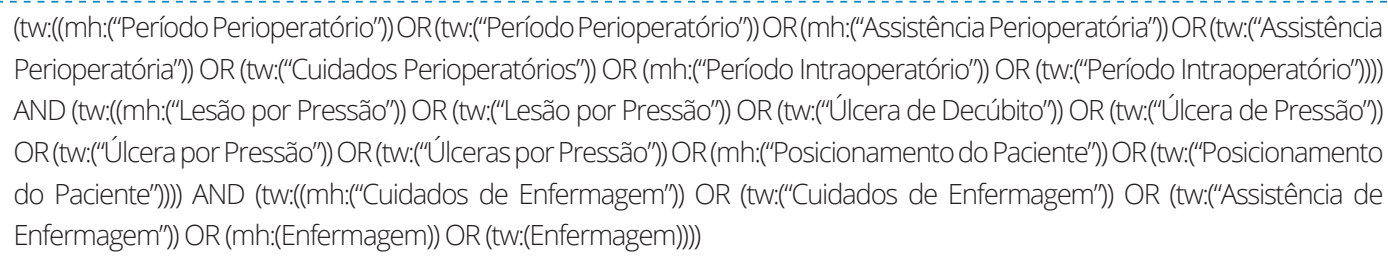 \\
\hline
\end{tabular}

DC (descritor controlado); DNC (descritor não controlado). 
nos idiomas inglês, português e espanhol, relacionados à temática de investigação e sem delimitação de recorte temporal. Excluíram-se notas, monografias, dissertações e teses.

O levantamento bibliográfico foi realizado no mês de janeiro de 2020 nas bases eletrônicas de dados Web of Science, Medical Literature Analysis and Retrieval System Online (MEDLINE via PubMed), Cumulative Index to Nursing and Allied Health Literature (CINAHL-Ebsco), Cochrane, Scopus e índice bibliográfico LILACS (Literatura LatinoAmericana em Ciências da Saúde via BVS).

Os descritores controlados e não controlados foram selecionados por meio de consulta aos termos do Medical Subject Headings (MeSH), Descritores em Ciências da Saúde (DeCS) e List of Headings do CINAHL Information Systems. As expressões de buscas foram elaboradas utilizando os operadores booleanos “OR” "AND”. Optou-se por diferentes estratégias de buscas, devido às peculiaridades das bases e do índice. A sintaxe das buscas está descrita na Tabela 1.

$\mathrm{O}$ acesso às produções ocorreu por meio do Portal de Periódicos da Coordenação de Aperfeiçoamento de Pessoal de Nível Superior (CAPES). Com o intuito de minimizar prováveis erros ou vieses de aferição dos estudos, a seleção foi desenvolvida por dois revisores, independentemente, em duas etapas. $\mathrm{Na}$ primeira etapa, realizou-se a leitura de título e resumo e, na segunda, fez-se a leitura do texto completo. Nos casos em que ocorreram desacordos, houve discussão entre os dois avaliadores para alcançar um consenso.

A busca resultou em 288 produções. Ressalta-se que os artigos duplicados em mais de uma base de dados ou índice foram contabilizados apenas uma vez, sendo 33 removidos por duplicatas. Assim, na primeira etapa, 255 artigos foram selecionados para a leitura de título e resumo. Após a aplicação dos critérios de inclusão e exclusão, 240 artigos foram descartados (15 eram revisões de literatura, 183 não estavam relacionados à temática, 27 eram notas e 15 eram monografias, dissertações ou teses). $\mathrm{Na}$ segunda etapa, 15 artigos foram elegíveis para a leitura de texto completo, sendo excluídas três produções por não abordarem a temática de investigação, restando 12 artigos, os quais compuseram a amostra e foram analisados. A Figura 1 descreve o fluxograma dos artigos selecionados.

Os dados foram extraídos mediante formulário contendo informações sobre identificação do artigo, país e ano de publicação, tipo de estudo, amostra, intervenção, principais resultados e nível de evidência dos estudos. Para esta análise,
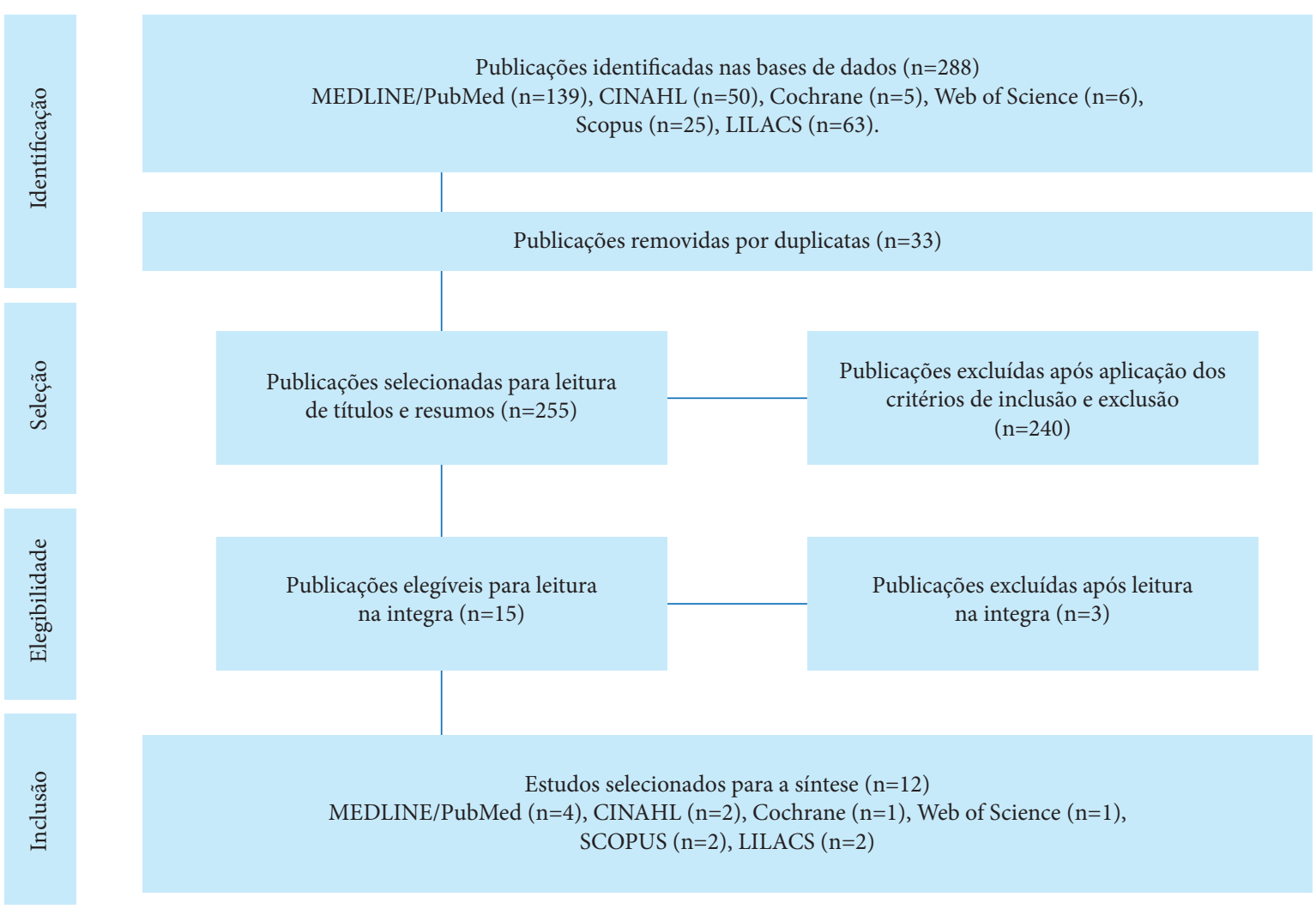

Figura 1. Fluxograma de identificação, seleção, elegibilidade e inclusão dos artigos. Teresina (PI), Brasil, 2020. 
classificaram-se as evidências, conforme o modelo proposto por Melnyk e Fineout-Overholt ${ }^{12}$ : nível I - evidências oriundas de revisão sistemática ou metanálise de todos ensaios clínicos relevantes randomizados controlados ou provenientes de diretrizes clínicas baseadas em revisões sistemáticas de ensaios clínicos randomizados controlados; nível II - evidências derivadas de pelo menos um ensaio clínico randomizado controlado bem delineado; nível III - evidências obtidas de ensaios clínicos bem delineados sem randomização; nível IV - evidências provenientes de estudos de coorte e de caso-controle bem delineados; nível $\mathrm{V}$ - evidências originárias de revisão sistemática de estudos descritivos e qualitativos; nível VI - evidências derivadas de um único estudo descritivo ou qualitativo; e nível VII evidências oriundas de opinião de autoridades e/ou relatórios de comitês de especialistas.

\section{RESULTADOS}

A síntese dos achados foi organizada na Tabela 2 e a análise crítica possibilitou a organização dos resultados por similaridade semântica, o que permitiu a construção de duas categorias: intervenções tecnológicas e educativas.

Quanto ao país de publicação, o Brasil se destacou com quatro produções. Com relação ao tipo de estudo, seis artigos são estudos de coorte, com nível de evidência IV. A principal intervenção foi os protocolos para prevenção de LP, com três publicações. Ademais, identificou-se que as intervenções foram efetivas na redução da incidência de LP em pacientes cirúrgicos em todos os estudos selecionados.

\section{DISCUSSÃO}

\section{Intervenções tecnológicas}

Intervenções aplicadas na prevenção de LP relacionam-se diretamente ao alívio de pressões durante e após a permanência do paciente sobre o colchão-padrão da mesa cirúrgica. Para tanto,é necessária a implementação de medidas preventivas nas fases perioperatórias, ou seja, no pré-, intra- e pós-operatório ${ }^{24}$.

Nesse âmbito, escalas para avaliar o risco de lesões de pele no centro cirúrgico devem ser aplicadas. Vale ressaltar que a escala de Braden não avalia de maneira específica os fatores de risco em pacientes cirúrgicos, tais como a duração da cirurgia, visto que uma hora de cirurgia é capaz de aumentar em 1,07\% o risco para desenvolvimento de $\mathrm{LP}^{17,25}$.

Escalas intraoperatórias específicas que avaliam o risco de lesões decorrentes do posicionamento cirúrgico permitem o reconhecimento mais efetivo dos fatores de risco e contribuem para a elaboração do plano de cuidado individualizado, o que garante uma assistência perioperatória de qualidade e com segurança. Dessa forma, surgiu a escala de Munro, a primeira específica na identificação do risco de desenvolvimento de LP em pacientes cirúrgicos, a qual possui três seções: pré-operatória (mobilidade e índice de massa corporal), intraoperatória (pontuação da American Society of Anesthesiologists, ASA, e temperatura corporal) e pós-operatória (duração da cirurgia e perdas sanguíneas), em que o nível de risco é pontuado para cada fase, obtendo-se uma pontuação acumulativa ao final ${ }^{26}$.

Outra ferramenta identificada foi a classificação de risco Scott Triggers, a qual avalia a idade do paciente, os níveis de albumina ou IMC, os índices recomendados pela ASA e a duração estimada de cirurgia, recomendada pela Associação de Enfermeiros Perioperatórios dos Estados Unidos. No Brasil, foi desenvolvida e validada a escala de avaliação do risco para o desenvolvimento de lesões decorrentes do posicionamento cirúrgico do paciente (ELPO), cujo escore varia de 7 a 35 pontos, sendo que quanto maior o escore, maior o risco para desenvolver lesões ${ }^{17,20}$.

A ELPO possui sete itens: tipo de posição cirúrgica, tempo de cirurgia, tipo de anestesia, superfície de suporte, posição dos membros, comorbidades e idade do paciente. Por ser um instrumento válido e confiável para avaliar o risco de desenvolvimento de lesões decorrentes do posicionamento cirúrgico, a aplicação da ELPO pode auxiliar na tomada de decisões do enfermeiro, garantindo a melhoria da assistência de enfermagem, a segurança do paciente e a redução de LPs'1.

Para que as intervenções sejam efetivas, são necessários dispositivos eficazes na prevenção desse tipo de lesão. Assim, podem ser utilizadas superfícies de apoio e estruturas especializadas, como revestimentos, estofamentos e sistemas integrados para redistribuição do peso, com o intuito de controlar a pressão, o cisalhamento e o atrito dos tecidos, mantendo o microclima e outras funções terapêuticas ${ }^{27}$.

A cama Clinitron Rite Hite foi uma estratégia utilizada no pós-operatório em que o paciente repousa em um colchão de ar de silício fluidizado, o qual reduz o cisalhamento, a maceração, a fricção e a umidade da pele. Obteve-se um resultado positivo, pois no universo de 28 pacientes, 
Tabela 2. Síntese das produções incluídas abordando identificação, ano, país, tipo de estudo, amostra, nível de evidência, intervenção e principais resultados. Teresina (PI), Brasil, 2020.

\begin{tabular}{|c|c|c|c|c|}
\hline Artigo & $\begin{array}{l}\text { Ano e } \\
\text { país }\end{array}$ & $\begin{array}{l}\text { Tipo de estudo, amostra e nível } \\
\text { de evidência }\end{array}$ & Intervenção & Principais resultados \\
\hline $\mathrm{A} 1^{13}$ & $\begin{array}{l}2011 \\
\text { EUA }\end{array}$ & $\begin{array}{c}\text { Estudo de coorte; } 25 \text { pacientes } \\
\text { submetidos a cirurgia cardíaca; } \\
\text { nível IV }\end{array}$ & Cama Clinitron Rite Hite & $\begin{array}{l}\text { Reduziu o número de pacientes } \\
\text { que desenvolveram LP. Não houve } \\
\text { progresso no estágio de lesões } \\
\text { anteriormente desenvolvidas. }\end{array}$ \\
\hline $\mathrm{A} 2^{14}$ & $\begin{array}{l}\text { 1998, } \\
\text { Reino } \\
\text { Unido }\end{array}$ & $\begin{array}{l}\text { Ensaio clínico randomizado } \\
\text { controlado; } 246 \text { pacientes } \\
\text { cirúrgicos da clínica vascular e } \\
\text { ginecológica; nível II }\end{array}$ & $\begin{array}{l}\text { Colchão de polímero } \\
\text { viscoelástico seco }\end{array}$ & $\begin{array}{l}\text { Reduziu a pressão sobre } \\
\text { proeminências ósseas } \\
\text { e, consequentemente, o } \\
\text { aparecimento de lesões. }\end{array}$ \\
\hline$A 3^{15}$ & $\begin{array}{l}2001 \\
\text { EUA }\end{array}$ & $\begin{array}{l}\text { Estudo randomizado controlado; } \\
324 \text { pacientes submetidos a } \\
\text { cirurgias de grande porte; nível II }\end{array}$ & Terapia com cobertores aquecidos & $\begin{array}{c}\text { Reduziu as taxas de } \\
\text { desenvolvimento de LP, } \\
\text { promovendo adequada circulação } \\
\text { sanguínea e oxigenação dos } \\
\text { tecidos. }\end{array}$ \\
\hline$A 4^{16}$ & $\begin{array}{l}2017 \\
\text { EUA }\end{array}$ & $\begin{array}{l}\text { Estudo de coorte; } 183 \text { pacientes } \\
\text { submetidos a traqueostomia; nível }\end{array}$ & $\begin{array}{l}\text { Protocolo de cuidados para } \\
\text { prevenção de LP em traqueostomia }\end{array}$ & $\begin{array}{c}\text { Reduziu a incidência de LPS } \\
\text { decorrentes do tubo de } \\
\text { traqueostomia. }\end{array}$ \\
\hline$A 5^{17}$ & $\begin{array}{l}\text { 2019, } \\
\text { Brasil }\end{array}$ & $\begin{array}{l}\text { Estudo de coorte; } 278 \text { pacientes } \\
\text { submetidos a intervenções } \\
\text { cirúrgicas eletivas; nível IV }\end{array}$ & $\begin{array}{c}\text { Escala de avaliação de risco para } \\
\text { o desenvolvimento de lesões } \\
\text { decorrentes do posicionamento } \\
\text { cirúrgico (ELPO) }\end{array}$ & $\begin{array}{l}\text { Identificou os fatores de riscos } \\
\text { para desenvolvimento de lesões } \\
\text { decorrentes do posicionamento } \\
\text { cirúrgico. }\end{array}$ \\
\hline$A 6^{18}$ & $\begin{array}{l}2012 \\
\text { EUA }\end{array}$ & $\begin{array}{l}\text { Estudo de coorte; } 424 \text { funcionários } \\
\text { de dois centros cirúrgicos; nível IV }\end{array}$ & $\begin{array}{l}\text { Protocolo de prevenção de lesões } \\
\text { por pressão }\end{array}$ & $\begin{array}{l}\text { Reduziu número de LPs em } \\
\text { 36\% através da implantação } \\
\text { de protocolo e intervenções } \\
\text { educativas. }\end{array}$ \\
\hline$A 7^{19}$ & $\begin{array}{l}\text { 2017, } \\
\text { Índia }\end{array}$ & $\begin{array}{l}\text { Estudo randomizado controlado; } \\
40 \text { funcionários de um centro } \\
\text { cirúrgico; nível II }\end{array}$ & Intervenção educativa & $\begin{array}{l}\text { Reduziu os fatores de riscos para } \\
\text { desenvolvimento de LP. }\end{array}$ \\
\hline$A 8^{20}$ & $\begin{array}{l}\text { 2019, } \\
\text { EUA }\end{array}$ & $\begin{array}{l}\text { Estudo de coorte; } 100 \text { pacientes de } \\
\text { um centro cirúrgico; nível IV }\end{array}$ & $\begin{array}{l}\text { Sobreposição de pressão alternada } \\
\text { de baixo perfil }\end{array}$ & $\begin{array}{l}\text { Nenhum dos pacientes } \\
\text { desenvolveu LP no perioperatório } \\
\text { usando essa terapia. }\end{array}$ \\
\hline A921 & $\begin{array}{l}\text { 2011, } \\
\text { Reino } \\
\text { Unido }\end{array}$ & $\begin{array}{l}\text { Estudo randomizado controlado; } \\
119 \text { pacientes foram recrutados } \\
\text { para o grupo-controle e } 120 \text { para o } \\
\text { grupo de intervenção; nível II }\end{array}$ & $\begin{array}{l}\text { Bota para minimizar a pressão no } \\
\text { calcanhar }\end{array}$ & $\begin{array}{l}\text { Avaliada por 59\% dos pacientes } \\
\text { como confortável, reduziu a } \\
\text { incidência de lesões no calcâneo. }\end{array}$ \\
\hline $\mathrm{A} 10^{22}$ & $\begin{array}{l}\text { 2018, } \\
\text { Brasil }\end{array}$ & $\begin{array}{l}\text { Estudo randomizado controlado; } \\
20 \text { pacientes usando superfícies de } \\
\text { apoio; nível II }\end{array}$ & Superfícies de apoio & $\begin{array}{l}\text { Regiões sacrais e calcâneas } \\
\text { sofreram menores pressões } \\
\text { usando o polímero viscoelástico. }\end{array}$ \\
\hline $\mathrm{A} 11^{23}$ & $\begin{array}{l}\text { 2017, } \\
\text { Brasil }\end{array}$ & $\begin{array}{c}\text { Estudo de coorte; } 359 \text { pacientes } \\
\text { submetidos a procedimentos } \\
\text { cirúrgicos robóticos urológicos; } \\
\text { nível IV }\end{array}$ & $\begin{array}{c}\text { Protocolo de prevenção de LP } \\
\text { no centro cirúrgico e simulação } \\
\text { realística }\end{array}$ & $\begin{array}{c}\text { Observou-se a efetividade do } \\
\text { protocolo no centro cirúrgico por } \\
\text { meio da atuação multiprofissional } \\
\text { integrada, preparando-os para a } \\
\text { avaliação adequada do risco para } \\
\text { lesões. }\end{array}$ \\
\hline $\mathrm{A} 12^{1}$ & $\begin{array}{l}\text { 2016, } \\
\text { Brasil }\end{array}$ & $\begin{array}{l}\text { Pesquisa metodológica; } 115 \\
\text { pacientes submetidos a } \\
\text { procedimentos cirúrgicos de } \\
\text { qualquer especialidade; nível VI }\end{array}$ & $\begin{array}{c}\text { Construção e validação da } \\
\text { escala de avaliação de risco para } \\
\text { o desenvolvimento de lesões } \\
\text { decorrentes do posicionamento } \\
\text { cirúrgico (ELPO) }\end{array}$ & $\begin{array}{l}\text { A ELPO mostrou-se um } \\
\text { instrumento válido e confiável } \\
\text { para a avaliação de risco de } \\
\text { desenvolvimento de lesões } \\
\text { decorrentes do posicionamento } \\
\text { cirúrgico em pacientes adultos. }\end{array}$ \\
\hline
\end{tabular}

Verificou-se que os artigos foram publicados entre 1998 e 2019, prevalecendo o ano de 2017 com três artigos. 
apenas um desenvolveu LP de estágio I. O custo com essa intervenção foi de US\$ 18.000 com 28 pacientes, bem inferior ao tratamento anterior, que foi de US\$ 9.134 por paciente ${ }^{13}$.

O colchão constituído por polímero viscoelástico seco, de uso intraoperatório, reduziu a pressão sobre as proeminências ósseas em $9 \%$ em relação ao colchão-padrão, além de minimizar o atrito e o cisalhamento. Entretanto estudos avaliaram as diferentes pressões nas proeminências ósseas das regiões occipital, subescapular, sacral e calcânea com superfícies de apoio contendo polímero viscoelástico, espumas seladas e espumas macias e demonstrou que as regiões sacrais e calcâneas, áreas de maior acometimento de LP, obtiveram maiores pressões sob o uso do polímero viscoelástico, o que destaca a necessidade de outras estratégias de prevenção associadas ${ }^{14,22}$.

O calcanhar é um dos locais mais comuns para o aparecimento de LP em centro cirúrgico, por isso foram projetados inúmeros dispositivos para diminuir a pressão nessa região, mas foi observado que os colchões de ar não são tão eficazes para prevenção de LP como aqueles específicos para calcanhar. Assim, um dispositivo em formato de bota foi projetado para reduzir a pressão do local, levantando o calcanhar com uma almofada presa por dois velcros e contendo na parte interna uma espuma no formato de "caixa de ovo". Não se observaram lesões decorrentes desse dispositivo e ele foi avaliado por $59 \%$ dos pacientes como confortável ${ }^{21}$.

Outra terapia preventiva bastante utilizada em países com frio extremo foi o uso de cobertores aquecidos, já que diminuem o risco de hipotermia relacionada à redução da oxigenação dos tecidos, prejudicando a integridade da pele. Essa estratégia reduziu o surgimento de LP em 46\% dos pacientes cirúrgicos ${ }^{24}$.

A sobreposição de pressão alternada de baixo perfil também é uma prevenção eficaz, uma vez que possui um sistema de suporte controlado por computador, o qual distribui o peso do paciente mediante pequenos pontos nodais de contato alternado, com insuflação e desinflação periódica, em ciclo de cinco minutos, em cada zona corporal, usada durante todo o procedimento cirúrgico. Identificou-se que nenhum dos pacientes usando essa terapia desenvolveu LP no perioperatório ${ }^{28}$.

Os pacientes que necessitam de traqueostomia frequentemente apresentam múltiplos fatores de risco para o desenvolvimento de LP decorrente do dispositivo, sendo importante a instalação de medidas preventivas. Estudo realizado nos Estados Unidos demostrou a eficácia de um curativo com hidrocoloide embaixo do flange do tubo traqueostomiano pós-operatório imediato, o qual diminuiu a incidência de LP de 10,93 para 1,29\% ${ }^{16}$.

\section{Intervenções educativas}

As intervenções educativas direcionadas para a equipe multidisciplinar atuante em centros cirúrgicos tiveram efeito positivo nos escores de prevenção de LP, principalmente no que se refere aos enfermeiros perioperatórios. Esses estão mais próximos aos pacientes e devem ser capacitados para a detecção dos fatores de riscos e gerenciamento do cuidado, apresentando conhecimento das diretrizes de posicionamento ${ }^{19,25,29}$.

A equipe deve ser treinada tanto para a implementação de escalas como de dispositivos para alívio de pressões, visto que, por meio dessas ações, é possível desenvolver habilidades técnicas e raciocínio crítico para analisar o melhor caminho a ser seguido, em consonância com as particularidades e especificidades dos procedimentos e dos riscos de cada paciente. Estudos verificaram que os enfermeiros do centro cirúrgico que receberam educação adicional apresentaram atitudes positivas com relação à competência, à prioridade e à responsabilidade pessoal para prevenção LP, destacando que o treinamento no ambiente de trabalho é imprescindível no enfrentamento desse agravo ${ }^{19,23,25}$.

Observou-se também em estudo realizado nos Estados Unidos que a instituição de um protocolo para prevenção de LP sem o treinamento prévio dos profissionais do centro cirúrgico não obteve êxito, pois reduziu apenas $10 \%$ o número de LPs. Porém, após o treinamento da equipe, o número de LPs diminuiu 36\%, reforçando que a educação continuada, associada à implementação de protocolos atualizados com treinamentos e pautados nas melhores evidências científicas é fundamental, pois reduziu as ações judiciais por negligência, bem como os números de morbidade e mortalidade ${ }^{18}$.

A simulação realística foi outra intervenção educativa para prevenção de LP no centro cirúrgico. Ressalta-se que os cenários simulados permitem a aproximação do profissional com a realidade, destacando-se como oportunidade para prever erros e evitá-los quando surgirem em situações semelhantes no futuro. Isso aumenta a segurança do profissional e do paciente cirúrgico por prevenir eventos e danos durante a cirurgia. Em um estudo em que participaram enfermeiros, técnicos de enfermagem, cirurgiões e anestesiologistas a simulação de posicionamento cirúrgico foi realizada previamente ao procedimento. Essa prática reduziu a incidência de LP 
em pacientes cirúrgicos para zero, configurando-se uma estratégia positiva quando aliada a um protocolo conciso e ao envolvimento da equipe interdisciplinar ${ }^{23}$.

Uma das limitações deste estudo foi a escassez de pesquisas relacionadas à prevenção de LP em pacientes cirúrgicos, pois, na maioria das produções encontradas, as intervenções estavam voltadas para prevenção de LP em outros cenários.

\section{CONCLUSÃO}

Identificou-se que as intervenções tecnológicas para prevenção de lesões por pressão no centro cirúrgico foram colchões para distribuição de pressão, dispositivo para redução de pressão no calcâneo, instrumentos validados específicos para a classificação do risco de LP em pacientes cirúrgicos, além de superfícies de apoio contendo polímero viscoelástico e outros utensílios para alívio da pressão decorrente do peso e de dispositivos médicos. Com relação às intervenções educativas, destacaram-se os protocolos, os treinamentos e a simulação realística. Observou-se que essas estratégias reduziram a incidência de LP, além de diminuírem os custos e garantirem a satisfação dos pacientes.

As intervenções tecnológicas e educativas, quando combinadas, mostraram-se efetivas na prevenção de LP no centro cirúrgico, o que enfatiza a importância da ampliação dessas estratégias nos serviços de saúde no intuito de minimizar esse agravo. Além do mais, sugere-se que estudos de elevada evidência científica acerca da temática sejam desenvolvidos, principalmente no cenário brasileiro, a fim de nortear boas práticas de enfermagem na prevenção de LP no período perioperatório.

\section{CONTRIBUIÇÃO DOS AUTORES}

Conceitualização; Bezerra, SMG, Brito JFP, Lira JAC, Barbosa NS, Carvalho KG e Sousa LS; Redação - Primeira Versão; Bezerra, SMG, Brito JFP, Lira JAC, Barbosa NS, Carvalho KG e Sousa LS; Redação - Revisão e Edição; Bezerra, SMG, Brito JFP e Lira JAC; Supervisão, Bezerra, SMG, Brito JFP, Lira JAC e Barbosa NS.

\section{REFERÊNCIAS}

1. Lopes CMM, Haas VJ, Dantas RAS, Oliveira CG, Galvão CM. Assessment scale of risk for surgical positioning injuries. Rev Latino-Am Enfermagem. 2016;24:e2704. https://doi. org/10.1590/1518-8345.0644.2704

2. Scott SM. Creating a strategic plan for perioperative pressure ulcer prevention. AORN J. 2016;103(4):P13-4. https://doi. org/10.1016/S0001-2092(16)30017-5

3. Cheever KH, Hinkle JL. Brunner \& Suddarth - Tratado de enfermagem médico-cirúrgica. Rio de Janeiro: Guanabara Koogan; 2016.

4. Brasil. Ministério da Saúde. Documento de referência para o Programa Nacional de Segurança do Paciente. Brasília: Ministério da Saúde; 2014. [citado em 2 Ago 2019]. Disponível em: http://bvsms.saude.gov.br/bvs/publicacoes/ documento_referencia_programa_nacional_seguranca.pdf

5. Engels D, Austin M, McNichol L, Fencl J, Gupta S, Kazi H. Pressure ulcers: factors contributing to their development in the OR. AORN J. 2016;103(3):271-81. https://doi. org/10.1016/j.aorn.2016.01.008

6. Donoso MTV, Barbosa SAS, Simino GPRS, Couto BRGM, Ercole FF, Barbosa JAG. Análise de custos do tratamento de lesão por pressão em pacientes internados. Rev enferm Cent Oeste Min. 2019; 9:e3446. https://doi.org/10.19175/ recom.v9i0.3446
7. Spruce L. Back to basics: preventing perioperative pressure injuries. AORN J. 2017; 105(1):92-9. https://doi.org/10.1016/j. aorn.2016.10.018

8. Menezes S, Rodrigues R, Tranquada R, Müller S, Gama K, Manso T. Injuries resulting from positioning for surgery: incidence and risk factors. Acta Med Port. 2013;26(1):12-6.

9. Paul R, McCutcheon SP, Tregarthen JP, Denend LT, Zenios SA. CE: Sustaining pressure ulcer best practices in a high-volume cardiac care environment. Am J Nurs. 2014;114(8):34-44. https://doi.org/10.1097/01.NAJ.0000453041.16371.16

10. Whittemore $R$, Knafl K. The integrative review: updated methodology. J Adv Nurs. 2005;52(5):546-53. https://doi. org/10.1111/j.1365-2648.2005.03621.x

11. Lockwood C, Porritt K, Munn Z, Rittenmeyer L, Salmond S, Bjerrum M, et al. Chapter 2: Systematic reviews of qualitative evidence. In: Aromataris E, Munn Z (Eds.). Joanna Briggs Institute Reviewer's Manual. Adelaide: The Joanna Briggs Institute; 2017.

12. Melnyk BM, Fineout-Overholt E. Making the case for evidence-based practice. In: Evidence-based practice in nursing \& healthcare: a guide to best practice. Philadelphia: Lippincot Williams \& Wilkins; 2005. https://doi.org/10.1037/ t68160-000 
13. Jackson M, McKenney T, Drumm J, Merrick B, LeMaster T, VanGilder C. Pressure ulcer prevention in high-risk postoperative cardiovascular patients. Crit Care Nurse. 2019;31(4):44-52.

14. Nixon J, McElvenny D, Mason S, Brown J, Bond S. A sequential randomised controlled trial comparing a dry visco-elastic polymer pad and standard operating table mattress in the prevention of post-operative pressure sores. Int J Nurs Stud. 1998;35(4):193-203. https://doi.org/10.1016/S00207489(98)00023-6

15. Scott EM, Leaper DJ, Clark M, Kelly PJ. Effects of warming therapy on pressure ulcers - a randomized trial. AORNJ. 2001;73(5):92138. https://doi.org/10.1016/S0001-2092(06)61744-4

16. O'Toole TR, Jacobs N, Hondorp B, Crawford L, Lisa R, Boudreau LR, Jeffe J, et al. Prevention of tracheostomyrelated hospital-acquired pressure ulcers. Otolaryngol Head Neck Surg. 2017;156(4):642-51. https://doi. org/10.1177/0194599816689584

17. Peixoto CA, Ferreira MBG, Felix MMS, Pires PS, Barichello E, Barbosa $\mathrm{MH}$. Risk assessment for perioperative pressure injuries. Rev Latino-Am Enfermagem. 2019;27:e3117. https://doi.org/10.1590/1518-8345.2677-3117

18. Strasser LA. Improving skin integrity in the perioperative environment using an evidence-based protocol. Dermatol Nurs. 2012;4(6):351-60. https://doi.org/10.1097/ JDN.0b013e318274b46c

19. Thenmozhi N, Valliammal B, Kiruba JA. A descriptive study to assess the adequacy of nursing measures carried out to minimize the pressure ulcers during peri-operative period. Int J Nurs Educ. 2017;(9)3:99-102. https://doi. org/10.5958/0974-9357.2017.00077.0

20. Oliveira KF, Rodrigues LP, Barichello E, Chavaglia SRR, Cunha $D F$, Ferreira MBG, et al. Bioimpedance as an indicator in the distribution of interface pressure in vulnerable regions for pressure ulcers: a preliminary study. Int J Nurs Pract. 2019;25(4):e12738. https://doi.org/10.1111/ijn.12738

21. Donnelly J, Winder J, Kernohan WG, Stevenson M. An $\mathrm{RCT}$ to determine the effect of a heel elevation device in pressure ulcer prevention post-hip fracture. J Wound Care. 2011;20(7):309-18. https://doi.org/10.12968/ jowc.2011.20.7.309

22. Oliveira KF, Pires PS, De-Mattia AL, Barichello E, Galvão CM, Araújo CA, et al. Influence of support surfaces on the distribution of body interface pressure in surgical positioning. Rev Latino-Am Enfermagem. 2018;26:e3083. https://doi.org/10.1590/1518-8345.2692.3083

23. Angelo CS, Pachioni CFM, Joaquim EHG, Silva EAL, Santos $\mathrm{GG}$, Bonfim IM, et al. Efetividade do protocolo prevenção de lesões de pele em cirurgias urológicas robóticas. Rev SOBECC. 2017;22(3):152-60.

24. Scott SM. Progress and challenges in perioperative pressure ulcer prevention. J Wound Ostomy Cont. 2015;42(5):480-5. https://doi.org/10.1097/WON.0000000000000161

25. Putnam K. Minimizing pressure ulcer risk for surgical patients. AORN J. 2016;103(4):P7-9. https://doi.org/10.1016/ S0001-2092(16)30009-6

26. Munro CA. The development of a pressure ulcer risk-assessment scale for perioperative patients. AORN J. 2010;92(3):272-87. https://doi.org/10.1016/j. aorn.2009.09.035

27. Yoshimura M, Ohura N, Tanaka J, Ichimura S, Kasuya Y, Hotta O. et al. Soft silicone foam dressing is more effective than polyurethane film dressing for preventing intraoperatively acquired pressure ulcers in spinal surgery patients: the Border Operating room Spinal Surgery (BOSS) trial in Japan. Int Wound J. 2018;15(2):188-97. https://doi.org/10.1111/ iwj.12696

28. Joseph J, McLaughlin D, Darian V, Hayes L, Siddiqui A. Alternating pressure overlay for prevention of intraoperative pressure injury. J Wound Ostomy Continence Nurs. 2019;46(1):13-7. https://doi.org/10.1097/ WON.0000000000000497

29. Ünver S, Fındık ÜY, Özkan ZK, Sürücü Ç. Attitudes of surgical nurses towards pressure ulcer prevention. J Tissue Viability. 2017;26(4):277-8. https://doi.org/10.1016/j.jtv.2017.09.001 